\title{
Molecular medicine and its impact on practice
}

\author{
David E Neal
}

Molecular medicine has had its greatest impact in the field of urologic oncology. Evolution of cancer cells has endowed them with the ability to evade mechanisms that control cell division, death and senescence. Disruption of interactions between stroma and epithelium results in angiogenesis, and cancer cells can penetrate blood and lymphatic vessels and metastasize. Recent identification of the mechanisms that underpin these abnormalities has depended on remarkable advances. These include cloning and amplification of DNA and RNA, high-throughput techniques for measuring nucleotides (the genome) and proteins (the proteome), and bioinformatics. Potential therapeutic targets are validated by downregulating the messenger RNA that encodes particular proteins using small-interfering RNA ('gene silencing') or by overexpressing genes. Transgenic animals that overexpress particular genes in specific tissues, or in which those genes are 'knocked out', have also helped.

There are now several examples of patients being treated successfully solely because of such advances. Imatinib (Gleevec/Glivec ${ }^{\circledR}$; Novartis, Camberley, UK) is used in chronic myeloid leukemia (CML) or gastrointestinal stromal tumors (GIST). Use of this drug is based on the finding that molecular alteration of a tyrosine kinase is the primary initiating event in these malignancies. In CML, this alteration results from the activating $B C R / A B L$ translocation and in GIST from overexpression of the $c-K I T$ oncogene; imatinib blocks both processes (and platelet-derived growth factor). It produces remarkable remissions even in pretreated patients. High-throughput techniques are beginning to identify alterations in other tyrosine kinases, and other specific small-molecule inhibitors such as imatinib will be found.

If, however, these alterations occur late in pathogenesis then the spectacular results seen with imatinib in CML may not be replicated. A feature of hormone-resistant breast cancers

\section{Advances in \\ molecular \\ medicine \\ are being \\ translated \\ into clinical \\ results... \\ academic \\ urology [must \\ be] fully \\ involved in \\ the design of \\ translational \\ trials}

DE Neal is a member of the Advisory Board of Nature Clinical Practice Urology and Professor of Surgical Oncology in the Department of Urology at Addenbrookes Hospital, Cambridge, UK.

\section{Competing interests}

The author declared he has no competing interests.

www.nature.com/clinicalpractice doi: $10.1038 /$ ncpuro0098 is overexpression of c-erbB2, a cell surface growth-factor receptor that is also a tyrosine kinase. Trastuzumab (Herceptin ${ }^{\circledR}$; Roche, Welwyn Garden City, UK) blocks this receptor and is now routinely used to treat patients with advanced breast cancer. Good results with gefitinib (Iressa ${ }^{\circledR}$; AstraZeneca, Alderley Park, UK), a small-molecule inhibitor of the epidermal growth factor receptor (EGFR), have also been reported in some patients with non-small-cell lung cancer ( $10 \%)$ who have mutations in the tyrosine kinase domain of EGFR.

Urologists know that renal cell cancers (RCCs) are vascular. How do tumours do this? The answer came from studies of von Hippel Lindau (VHL) disease which, in addition to tumor and cyst formation, is characterized by angiogenesis in the central nervous system and kidney. The product of the VHL gene on chromosome $3 p$ inhibits an oxygen sensor protein (hypoxia inducible factor $1 a$ [HIF1a]). When tissue is hypoxic, HIF1a upregulates vascular endothelial growth factor, which stimulates angiogenesis. In spontaneous RCCs, deletion or mutation of the VHL gene impairs its inhibitory function; hence the remarkable vascularity of these malignancies. Patients with advanced RCCs have had good clinical responses to novel angiogenesis inhibitors.

There are other drugs in the pipeline that block important, disease-related molecular targetssuch as mammalian target of rapamycin-that are being tested. The remarkable advances in molecular medicine are being translated into clinical results. We shall see many being used for the benefit of urologic patients over the forthcoming decade. During this period we must ensure that academic urology is fully involved in the design of translational trials. The goals for academic urology are clear; to ensure that good clinical scientists receive appropriate training, and that once trained, they can be retained by provision of appropriately attractive paths for career progression. 